\title{
A LEMMA IN TRANSCENDENTAL NUMBER THEORY
}

\author{
BY \\ ROBERT SPIRA
}

In this paper, we discuss a faulty lemma of Gelfond of use in the theory of algebraic independence of transcendental numbers. We then replace Gelfond's lemma by a clearly stated simpler lemma which is much easier to apply.

Gelfond's [1, p. 140] lemma is:

"LEMMA. Suppose $p, q, p>q^{\gamma}, r, r_{1}$ are positive rational integers, $\varepsilon>0$ and $\gamma$ are fixed, and all the numbers $\alpha_{1}, \alpha_{2}, \ldots, \alpha_{q}$, as well as the numbers $\beta_{1}, \beta_{2}, \ldots, \beta_{r}$ are distinct and arranged in order of increasing absolute values, in other words, $\left|\alpha_{k}\right|$ $\leqq\left|\alpha_{k+1}\right|$ and $\left|\beta_{k}\right| \leqq\left|\beta_{k+1}\right|$. We set $\left|\alpha_{q}\right|=\alpha,\left|\beta_{r}\right|=\beta$ and suppose that there exist constants $\gamma_{0}>0, \gamma_{1}>0, \gamma_{0}+\gamma_{1}<1$, such that $\alpha<(p q)^{\gamma_{1}}, \beta<(p q)^{\gamma_{0}}$. We also suppose that there exists a constant $\gamma_{2}$, such that the inequalities

$$
\begin{gathered}
\prod_{k=1: k \neq i}^{q}\left|\alpha_{i}-\alpha_{k}\right|>\exp \left(-\gamma_{2} q \ln p q\right), \\
\left|\alpha_{i}-\alpha_{k}\right|>\exp \left(-\gamma_{2} q \ln p q\right), \quad 1 \leqq i \leqq q, 1 \leqq k \leqq q
\end{gathered}
$$

are satisfied. Further, if the function $f(z)$ has the form

$$
f(z)=\sum_{k=0}^{p-1} \sum_{s=1}^{q} A_{k, s} z^{k} e^{z \alpha_{s}}
$$

where the numbers $A_{k, s}$ are not all zero, then at least one of the numbers

$$
\begin{aligned}
f^{(s)}\left(\beta_{k}\right), & 0 \leqq s \leqq r_{1}-1,1 \leqq k \leqq r, \\
r_{1} r \geqq[\lambda p q], & \lambda=\left(1+\gamma_{1}+2 \gamma_{2}+\varepsilon\right) /\left(1-\gamma_{1}-\gamma_{0}\right)
\end{aligned}
$$

is different from zero for sufficiently large pq."

Smelev [2] also makes use of this result.

We next discuss Gelfond's lemma.

First of all, the phrase "the numbers $A_{k, s}$ are not all zero," in the original is literally "the numbers $A_{k, s}$ in the aggregate are different from zero." This phrase is repeatedly mistranslated in [1] as "all different from zero."

Next one gets the impression that $p$ and $q$ are independent integer variables subject to $p>q^{\gamma}$. This condition is also written elsewhere as $p<q^{\gamma}$. The theorem as

Received by the editors April 7, 1969 and, in revised form, July 7, 1969.

Copyright (C) 1969, American Mathematical Society 
it stands does not say whether $\gamma$ is positive or negative, and the only place where " $\gamma$ " appears in the proof is in the statement "The condition $p>q$, appearing in the formulation of the lemma serves only to simplify the proof and may be replaced by a much weaker condition." The proof given fails at several points, and it is hard to see how to make use of either of the conditions, though both hold with different $\gamma$ 's, in the various applications. Perhaps the explanation for this peculiar condition is that in one of the uses of the lemma, the general condition with which we replace $p<q^{\gamma}$ reduces to a condition where $p<q^{\gamma}$ can be used.

One also gets the impression that either $r$ or $r_{1}$ is running, depending on the size of $p q$. Indeed, $r$ must be running, as otherwise the condition of the existence of $\gamma_{0}$ such that $\left|\beta_{r}\right|<(p q)^{\gamma_{0}}$ would be trivial. However, the lemma could be interpreted as having $r$ fixed. The quantity $q$ is running, so apparently we are dealing with a sequence of $\alpha_{j}$ 's. When the lemma is used it turns out that we are not dealing with a sequence of $\alpha_{j}$ 's, nor with a sequence of $\beta_{j}$ 's.

It appears that there are hidden assumptions which are intuitively verified when using the result.

We now give a lemma which will replace the one above. It also omits the condition of the existence of $\gamma_{2}$ and the associated conditions on the $\alpha_{j}$ 's. Further, it decreases $\lambda$, and we note that Gelfond states that this can be done. The new lemma adds conditions, conditions (iii) and (iv), on the rate of growth of the parameter $r_{1}$. In the applications, one has $r_{1}$ bounded or with a known rate of growth. In Gelfond's "proof", the author believes Gelfond is implicitly assuming a small rate of growth for $r_{1}$, as he appears to be abstracting from two different situations where this occurs. Our omission of the constant $\gamma_{2}$ and the associated conditions does not depend on the assumptions about $r_{1}$. This improvement of the lemma is due to a different method of estimation of the full Hermite interpolating polynomial.

Lemma. Let $N$ be a positive integer variable. Let $p=p(N), q=q(N), r=r(N)$ be positive integer functions of $N$ such that $p \cdot q=m$ tends to $\infty$ with $N$. To each $q=q(N)$ let there correspond a set $A_{q}$ of $q$ numbers $\alpha_{q, 1}, \alpha_{q, 2}, \ldots, \alpha_{q, q}$, which we write also by an abuse of notation as $\alpha_{1}, \ldots, \alpha_{q}$. To each $r=r(N)$ let there correspond a set $B_{r}$ of $r$ numbers $\beta_{r, 1}, \ldots, \beta_{r, r}$ which we also write as $\beta_{1}, \ldots, \beta_{r}$. We assume also that there exist positive constants $\gamma_{0}$ and $\gamma_{1}$ such that $\gamma_{0}+\gamma_{1}<1$, and for $p, q$, and $r$ (determined by $N$ ),

(i) $\left|\beta_{r, j}\right|<(p \cdot q)^{\gamma_{0}}$,

(ii) $\left|\alpha_{q, j}\right|<(p \cdot q)^{\gamma_{1}}$.

Let $\varepsilon$ be given, $\varepsilon>0$. Define

$$
\lambda=\left(1+\gamma_{1}+\varepsilon\right) /\left(1-\gamma_{1}-\gamma_{0}\right), \quad r_{1}=\text { the least integer } \geqq[\lambda p q] / r,
$$

and assume finally, that

(iii) $r_{1} ! \leqq m^{\gamma_{1} m}$, for $N$ sufficiently large, and

(iv) $r r_{1}=O(m)$. 
Then there is an $N_{0}$, such that for any particular $N$ greater than $N_{0}$, if the function $f(z)$ has the form

$$
f(z)=\sum_{k=0}^{p-1} \sum_{s=1}^{q} A_{k, s} z^{k} e^{z \alpha_{s}},
$$

where not all $A_{k, s}$ are zero, and if $r_{2} \geqq r_{1}$ then at least one of the numbers

$$
f^{(s)}\left(\beta_{k}\right), \quad 0 \leqq s \leqq r_{2}-1,1 \leqq k \leqq r
$$

is different from zero.

Proof. Without loss of generality, we can take $r_{2}=r_{1}$. We will arrive in the proof at various minima for $N_{0}$, and it will be understood that we take the largest of these. We set $\varepsilon=3 \delta$. Without loss of generality, we can take $\left|A_{k, s}\right| \leqq 1$, where at least one $A_{k, s}$ has absolute value 1 . For we can divide all the coefficients of $f(z)$ by the coefficient with largest absolute value. Arguing by contradiction, assume $N>N_{0}$, and $\lambda$ and $r_{1}$ given as above and that

$$
f^{(s)}\left(\beta_{k}\right)=0, \quad 0 \leqq s \leqq r_{1}-1,1 \leqq k \leqq r .
$$

Let $\Gamma$ be the circle $|z|=1$ and $\Gamma_{1}$ the circle $|\xi|=m^{1-\gamma_{1}}$. We take $N$ so large that $m \geqq 2$. Then $\Gamma$ is inside $\Gamma_{1}$. Now $\gamma_{0}+\gamma_{1}<1$, so $\gamma_{0}<1-\gamma_{1}$. Thus, the points $\beta_{j}$ lie in the interior of $\Gamma_{1}$, as $\left|\beta_{j}\right|<(p \cdot q)^{\gamma_{0}}=m^{\gamma_{0}}<m^{1-\gamma_{1}}$. Since $f^{(s)}\left(\beta_{k}\right)=0,0 \leqq s \leqq r_{1}-1$, $1 \leqq k \leqq r$, we have that

$$
f(\xi)=\left[\left(\xi-\beta_{1}\right)\left(\xi-\beta_{2}\right) \cdots\left(\xi-\beta_{r}\right)\right]^{r_{1}} g(\xi),
$$

where $g(\xi)$ is analytic everywhere.

Now, for $z$ on $\Gamma$, or indeed anywhere in the interior of $\Gamma_{1}$,

$$
\frac{1}{2 \pi i} \int_{\Gamma_{1}} \frac{g(\xi)}{\xi-z} d \xi=g(z)
$$

by the Cauchy integral formula, so

$$
f(z)=\left[\left(z-\beta_{1}\right)\left(z-\beta_{2}\right) \cdots\left(z-\beta_{r}\right)\right]^{r_{1}} \frac{1}{2 \pi i} \int_{\Gamma_{1}} \frac{1}{\left[\left(\xi-\beta_{1}\right) \cdots\left(\xi-\beta_{r}\right)\right]^{r_{1}}} \frac{f(\xi)}{\xi-z} d \xi,
$$

as we can divide out $\left[\left(\xi-\beta_{1}\right) \cdots\left(\xi-\beta_{r}\right)\right]^{r_{1}}$ in (1) for $\xi$ on $\Gamma_{1}$. Hence, again by the Cauchy integral formula

$$
f^{(s)}(0)=\frac{s !}{(2 \pi i)^{2}} \int_{\Gamma} \frac{1}{z^{s+1}}\left\{\int_{\Gamma_{1}}\left[\frac{\left(z-\beta_{1}\right) \cdots\left(z-\beta_{r}\right)}{\left(\xi-\beta_{1}\right) \cdots\left(\xi-\beta_{r}\right)}\right]^{r_{1}} \frac{f(\xi)}{\xi-z} d \xi\right\} d z .
$$

Now,

$$
\begin{aligned}
\left|f^{(s)}(0)\right| & \leqq \frac{s !}{(2 \pi)^{2}} 2 \pi \max _{|z|=1}\left|\int_{\Gamma_{1}}\left[\frac{\left(z-\beta_{1}\right) \cdots\left(z-\beta_{r}\right)}{\left(\xi-\beta_{1}\right) \cdots\left(\xi-\beta_{r}\right)}\right]^{r_{1}} \frac{f(\xi)}{\xi-z} a \xi\right| \\
& \leqq \frac{s !}{2 \pi} \max _{|z|=1}\left\{2 \pi m^{1-\gamma_{1}} \max _{|\xi|=m^{1-\gamma_{1}}}\left\{\left|\left[\frac{\left(z-\beta_{1}\right) \cdots\left(z-\beta_{r}\right)}{\left(\xi-\beta_{1}\right) \cdots\left(\xi-\beta_{r}\right)}\right]^{r_{1}} \frac{f(\xi)}{\xi-z}\right|\right\}\right\} .
\end{aligned}
$$


Also

$$
\begin{array}{rlrl}
|\xi-z| & \geqq|\xi|-|z|=m^{1-\gamma_{1}}-1, & \\
\left|\xi-\beta_{j}\right| \geqq|\xi|-\left|\beta_{j}\right| \geqq m^{1-\gamma_{1}}-m^{\gamma_{0}}, & j=1, \ldots, r, \\
\left|z-\beta_{j}\right| \leqq 1+\left|\beta_{j}\right| \leqq 1+m^{\gamma_{0}}, & j=1, \ldots, r .
\end{array}
$$

Thus,

$$
\left|f^{(s)}(0)\right| \leqq s ! m^{1-\gamma_{1}}\left[\frac{1+m^{\gamma_{0}}}{m^{1-\gamma_{1}}-m^{\gamma_{0}}}\right]^{r r_{1}} \frac{1}{m^{1-\gamma_{1}-1}} \max _{\mid \xi !=m^{1-\gamma_{1}}}|f(\xi)| .
$$

Next, we have

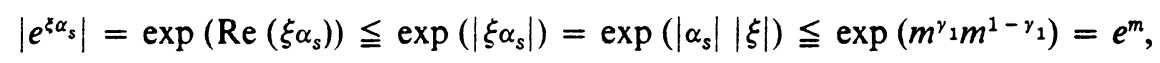

so

$$
\begin{aligned}
\max _{|\xi|=m^{1-\gamma_{1}}}|f(\xi)| & \leqq \max _{|\xi|=m^{1-\gamma_{1}}} \sum_{k=0}^{p-1} \sum_{s=1}^{q}|\xi|^{k} e^{m} \\
& \leqq p \cdot q\left(m^{1-\gamma_{1}}\right)^{p-1} e^{m} \\
& \leqq m \cdot e^{m} \cdot m^{m\left(1-\gamma_{1}\right)} .
\end{aligned}
$$

Thus, from (3) and (4) we obtain

$$
\begin{aligned}
\left|f^{(s)}(0)\right| & \leqq s ! \frac{m^{1-\gamma_{1}}}{m^{1-\gamma_{1}-1}} m^{r r_{1}\left(\gamma_{0}+\gamma_{1}-1\right)}\left[\frac{1+m^{-\gamma_{0}}}{1-m^{\gamma_{0}+\gamma_{1}-1}}\right]^{r r_{1}} m e^{m} m^{m\left(1-\gamma_{1}\right)} \\
& \leqq r_{1} ! m^{-\gamma_{1} m} m^{m} m^{-\lambda\left(1-\gamma_{0}-\gamma_{1}\right) m} \cdot A \\
& \leqq m^{m} m^{-\lambda\left(1-\gamma_{0}-\gamma_{1}\right) m} \cdot A,
\end{aligned}
$$

using (iii), where

$$
A=m e^{m}\left[\frac{1+m^{-\gamma_{0}}}{1-m^{\gamma_{0}+\gamma_{1}-1}}\right]^{r r_{1}} m^{\left(1-\gamma_{0}-\gamma_{1}\right)\left(\lambda m-r r_{1}\right)} .
$$

To obtain our first main result (7) below, we need only show $A \leqq m^{\delta m}$ for $N$ sufficiently large. Now, $\lambda m<[\lambda m]+1 \leqq r r_{1}$, since $r_{1} r$ is the least multiple of $r \geqq[\lambda m]$, unless $[\lambda m]=r_{1} r$, in which case $\lambda m-r r_{1}<1$. In either case,

$$
m^{\left(1-\gamma_{0}-\gamma_{1}\right)\left(\lambda m-r r_{1}\right)} \leqq m^{1-\gamma_{0}-\gamma_{1}} .
$$

Let $G(m)$ be a positive function of $m$. We have $G(m) \leqq m^{\varepsilon_{1} m}$ for $m$ sufficiently large for any given $\varepsilon_{1}>0$ if and only if $\varepsilon_{1} \geqq(\log G(m)) /(m \log m)$ for $m$ sufficiently large. Thus, if $\lim _{m \rightarrow \infty}(\log G(m)) /(m \log m)=0$, then we can conclude that we can assign $\varepsilon_{1}$ as we please, and then take $m$ beyond some fixed lower bound and have $G(m) \leqq m^{\varepsilon_{1} m}$.

Thus, if we take $G(m)$ successively as $m, e^{m}, m^{1-\gamma_{0}-\gamma_{1}}, 4^{K_{1} m}, K_{1}>0$, we obtain $m<m^{\delta m / 4}, e^{m}<m^{\delta m / 4}, m^{1-\gamma_{0}-\gamma_{1}}<m^{\delta m / 4}, 4^{K_{1} m}<m^{\delta m / 4}$, for $m$ sufficiently large. Finally, if $m$ is sufficiently large, $1+m^{-\gamma_{0}}<2$ and $1 /\left(1-m^{\gamma_{0}+\gamma_{1}-1}\right)<2$, so, using $r r_{1} \leqq K_{1} m$ from (iv), we obtain

$$
\left[\frac{1+m^{-\gamma_{0}}}{1-m^{\gamma_{0}+\gamma_{1}-1}}\right]^{r r_{1}} \leqq 4^{r r_{1}} \leqq 4^{K_{1} m}<m^{\delta m / 4}
$$

for $m$ sufficiently large. 
Thus, for $m$ sufficiently large, $A \leqq m^{\delta m}$, and we have

$$
\left|f^{(s)}(0)\right| \leqq m^{\left(1+\delta-\lambda\left(1-\gamma_{0}-\gamma_{1}\right)\right) m} \text {. }
$$

We note that (iv) could be replaced by

$$
\lim _{N \rightarrow \infty}\left(r r_{1} \log \left[\frac{1+m^{-y_{0}}}{1-m^{\gamma_{0}+y_{1}-1}}\right]\right) /(m \log m)=0 .
$$

We now apply the theory of full Hermite interpolation. Let $\alpha_{1}, \ldots, \alpha_{q}$ be distinct complex numbers and let $k_{1}, \ldots, k_{q}$ be positive integers and let $k_{1}+\cdots+k_{q}=k+1$. Let $y_{j}^{(v)}$ be constants, $j=1, \ldots, q, v=0,1, \ldots, k_{j}-1$. J. L. Walsh [3, pp. 49-50] gives a method of proof for the existence and uniqueness of a polynomial $P_{k}(z)$, of degree $\leqq k$, called the full Hermite interpolation polynomial, satisfying

$$
P_{k}^{(v)}\left(\alpha_{j}\right)=y_{j}^{(v)}, \quad j=1, \ldots, q ; \nu=0,1, \ldots, k_{j}-1 .
$$

I. S. Berezin and N. P. Zhidkov [4, pp. 145-147] construct such a polynomial, by first constructing polynomials $P_{i, j}(z)$ satisfying

$$
P_{i, j}^{(\nu)}\left(\alpha_{s}\right)=\delta_{i, s} \delta_{j, v}, \quad s=1, \ldots, q ; \nu=0,1, \ldots, k_{i}-1,
$$

where $\delta_{i, s}$ and $\delta_{j, v}$ are Kronecker deltas, so that

$$
P_{k}(z)=\sum_{i=1}^{q} \sum_{j=0}^{k_{i}-1} y_{i}^{(j)} P_{i, j}(z)
$$

Writing

$$
w(z)=\left(z-\alpha_{1}\right)^{k_{1}}\left(z-\alpha_{2}\right)^{k_{2}} \cdots\left(z-\alpha_{q}\right)^{k_{q}},
$$

they find

$$
P_{i, j}(z)=\left.\frac{w(z)}{\left(z-\alpha_{i}\right)^{k_{i}-j}} \frac{1}{j !} \sum_{n=0}^{k_{i}-j-1} \frac{1}{n !}\left(z-\alpha_{i}\right)^{n}\left[\frac{d^{n}}{d \xi^{n}}\left(\frac{\left(\xi-\alpha_{i}\right)^{k_{i}}}{w(\xi)}\right)\right]\right|_{\xi=\alpha_{i}} .
$$

In the special case of $k_{1}=k_{2}=\cdots=k_{q}=p$, we obtain

$$
P_{i, j}(z)=\left.\frac{w(z)}{\left(z-\alpha_{i}\right)^{p-j}} \frac{1}{j !} \sum_{n=0}^{p-j-1} \frac{1}{n !}\left(z-\alpha_{i}\right)^{n}\left[\frac{d^{n}}{d \xi^{n}}\left(\frac{\left(\xi-\alpha_{i}\right)^{p}}{w(\xi)}\right)\right]\right|_{\xi=\alpha_{i}},
$$

where $1 \leqq i \leqq q$ and $0 \leqq j \leqq p-1$, and $w(z)=\left[\left(z-\alpha_{1}\right) \cdots\left(z-\alpha_{q}\right)\right]^{p}$. Next, we write

so $C_{k}=P_{i, j}^{(k)}(0) / k$ !.

$$
P_{i, j}(z)=\sum_{k=0}^{m-1} C_{k} z^{k}
$$

Using (12), Gelfond now estimates $\left|C_{k}\right|$ by a complicated algebraic expansion of

$$
B_{i, n}=\left.\frac{d^{n}}{d \xi^{n}}\left(\frac{\left(\xi-\alpha_{i}\right)^{p}}{w(\xi)}\right)\right|_{\xi=\alpha_{i}},
$$

and arrives at his intricate conditions on the $\alpha_{j}$ 's. We proceed by an integral estimate. 
462

ROBERT SPIRE

[December

Let $\alpha=m^{\gamma_{1}}$. We have

$$
\begin{aligned}
C_{k} & =P_{i, j}^{(k)}(0) / k ! \\
& =\frac{1}{2 \pi i} \int_{|z|=2 \alpha}\left(\frac{w(z)}{\left(z-\alpha_{i}\right)^{p-j}} \frac{1}{j !} \sum_{n=0}^{p-j-1} \frac{1}{n !}\left(z-\alpha_{i}\right)^{n} B_{i, n}\right) \frac{d z}{z^{k+1}},
\end{aligned}
$$

so

$$
\left|C_{k}\right| \leqq 2 \alpha \max _{|z|=2 \alpha}\left|\frac{w(z)}{\left(z-\alpha_{i}\right)^{p-j}} \frac{1}{j !} \sum_{n=0}^{p-j-1} \frac{1}{n !}\left(z-\alpha_{i}\right)^{n} B_{i, n}\right| \cdot \frac{1}{(2 \alpha)^{k+1}} .
$$

Now, on $|z|=2 \alpha$, since $\left|\alpha_{j}\right| \leqq \alpha$, we have

$$
\alpha \leqq\left|z-\alpha_{j}\right| \leqq 3 \alpha,
$$

so

$$
\left|C_{k}\right| \leqq 2 \alpha(3 \alpha)^{q(p-1)}(3 \alpha)^{j} \frac{1}{j !}\left(\sum_{n=0}^{p-j-1} \frac{1}{n !}(3 \alpha)^{n}\left|B_{i, n}\right|\right)(2 \alpha)^{-1-k}
$$

Next,

$$
B_{i, n}=\frac{n !}{2 \pi i} \int_{|\xi|=2 \alpha} \frac{\left(\xi-\alpha_{i}\right)^{p}}{w(\xi)} \cdot \frac{1}{\left(\xi-\alpha_{i}\right)^{n+1}} d \xi
$$

so, using (13)

$$
\left|B_{i, n}\right| \leqq \frac{n !}{2 \pi} 4 \alpha \pi \frac{1}{\alpha^{q(p-1)}} \cdot \frac{1}{\alpha^{n+1}}=n ! \frac{2}{\alpha^{q(p-1)+n}} .
$$

Thus,

$$
\begin{aligned}
\left|C_{k}\right| & \leqq 2 \alpha(3 \alpha)^{q(p-1)}(3 \alpha)^{j} \frac{1}{j !}\left(\sum_{n=0}^{p-j-1}(3 \alpha)^{n} \frac{2}{\alpha^{q(p-1)+n}}\right) \frac{1}{(2 \alpha)^{k+1}} \\
& \leqq \frac{1}{(2 \alpha)^{k}} 3^{q(p-1)}(3 \alpha)^{p-1}\left(\sum_{n=0}^{p-j-1} 2 \cdot 3^{n}\right) \\
& \leqq 3^{q p} 3^{p} \alpha^{m}(p) \cdot 2 \cdot 3^{p-1} \\
& \leqq 2 \cdot p \cdot 3^{q p} 3^{2 p} \alpha^{m} \\
& \leqq m^{2} 3^{3 m} \alpha^{m}=m^{2} 3^{3 m} m^{\gamma_{1} m}
\end{aligned}
$$

and taking $G(m)=m^{2}$ respectively $3^{3 m}$, we obtain $m^{2}<m^{\delta m / 2}, 3^{3 m}<m^{\delta m / 2}$, for $m$ sufficiently large. Thus,

$$
\left|C_{k}\right| \leqq \exp \left(\left(\delta+\gamma_{1}\right) m \log m\right) .
$$

We next show that for $k$ and $r$ nonnegative integers, we have

$$
\left.\frac{d^{k}}{d z^{k}} z^{r} e^{\alpha z}\right|_{z=0}=\left.\frac{d^{r}}{d z^{r}} z^{k}\right|_{z=\alpha}
$$

The proof is as follows. For $r=0, k=0$, we have $e^{\alpha z}=1, z^{0}=1$. For $r=0, k>0$, we have

$$
\left.\frac{d^{k}}{d z^{k}} e^{\alpha z}\right|_{z=0}=\left.(\alpha)^{k} e^{\alpha z}\right|_{z=0}=\alpha^{k},\left.\quad z^{k}\right|_{z=\alpha}=\alpha^{k}
$$


so we have the result. For $k=0, r>0$, we have

$$
\left.\frac{d^{k}}{d z^{k}} z^{r} e^{\alpha z}\right|_{z=0}=\left.z^{r} e^{\alpha z}\right|_{z=0}=0,\left.\quad \frac{d^{r}}{d z^{r}} 1\right|_{z=\alpha}=0 .
$$

For $k=1, r>0$, we have

$$
\begin{gathered}
\left.\frac{d}{d z} z^{r} e^{\alpha z}\right|_{z=0}=r z^{r-1} e^{\alpha z}+\left.\alpha z^{r} e^{\alpha z}\right|_{z=0}=\left.r z^{r-1}\right|_{z=0}=1 \text { if } r=1, \\
=0 \text { otherwise, } \\
\begin{aligned}
\frac{d^{r}}{d z^{r}} z=1 & \text { if } r=1, \\
=0 & \text { otherwise. }
\end{aligned}
\end{gathered}
$$

Finally, let $k>1, r>0$, then

$$
\begin{aligned}
\left.\frac{d^{k}}{d z^{k}} z^{r} e^{\alpha z}\right|_{z=0} & =\left.\sum_{p=0}^{k}\left(\begin{array}{l}
k \\
p
\end{array}\right)\left(z^{r}\right)^{(p)} \alpha^{k-p} e^{\alpha z}\right|_{z=0}=r !\left(\begin{array}{l}
k \\
p
\end{array}\right) \alpha^{k-r} \quad \text { if } r \leqq k, \\
& =0 \quad \text { if } r>k ; \\
\left.\frac{d^{r}}{d z^{r}} z^{k}\right|_{z=\alpha} & =(k)(k-1) \cdots(k-r+1) \alpha^{k-r} \quad \text { if } r \leqq k, \\
& =0 \text { if } r>k,
\end{aligned}
$$

and the results are easily seen to be the same.

Hence, we obtain

$$
\begin{aligned}
P_{i, j}^{(r)}\left(\alpha_{s}\right) & =\left.\sum_{k=0}^{m-1} C_{k} \frac{d^{r}}{d z^{r}} z^{k}\right|_{z=\alpha_{s}} \\
& =\left.\sum_{k=0}^{m-1} C_{k} \frac{d^{k}}{d z^{k}} z^{r} e^{\alpha_{s} z}\right|_{z=0} .
\end{aligned}
$$

Thus,

$$
\begin{aligned}
A_{j, i} & =\sum_{r=0}^{p-1} \sum_{s=1}^{q} A_{r, s} P_{i, j}^{(r)}\left(\alpha_{s}\right) \\
& =\left.\sum_{r=0}^{p-1} \sum_{s=1}^{q} A_{r, s} \sum_{k=0}^{m-1} C_{k} \frac{d^{k}}{d z^{k}} z^{r} e^{\alpha_{s} z}\right|_{z=0} \\
& =\sum_{k=0}^{m-1} C_{k} f^{(k)}(0)
\end{aligned}
$$

Choose $A_{j, i}$ so $\left|A_{j, i}\right|=1$. Then from (7) and (14)

$$
\begin{aligned}
1 & \leqq \sum_{k=0}^{n-1}\left|C_{k}\right|\left|f^{(k)}(0)\right| \leqq m \exp \left[\left(\gamma_{1}+\delta+1+\delta-\lambda\left(1-\gamma_{0}-\gamma_{1}\right)\right) m \log m\right] \\
& =m \exp \left[\left(\gamma_{1}+1+2 \delta-\left(1+\gamma_{1}+\varepsilon\right)\right) m \log m\right] \\
& =m \exp (-\delta m \log m) .
\end{aligned}
$$

This is a contradiction for $m$ sufficiently large, thus proving the lemma. 


\section{REFERENCES}

1. A. O. Gelfond, Transcendental and algebraic numbers, Translated by L. F. Boron, Dover, New York, 1960.

2. A. A. Smelev, On the algebraic independence of certain transcendental numbers, Mat. Zametki 3 (1968), 51-58.

3. J. L. Walsh, Interpolation and approximation by rational functions in the complex plane, Amer. Math. Soc. Colloq. Publ., Vol. 20, Amer. Math. Soc., Providence, R. I., 1930.

4. I. S. Berezin and N. P. Zhidkov, Computing methods, Vol. I, Addison-Wesley, Reading, Mass., 1965.

Michigan State University,

East Lansing, Michigan 